\title{
Facile Impedimetric Analysis of Neuronal Exosome Markers in Parkinson's Disease Diagnostics
}

\author{
Ying Fu, ${ }^{\S}$ Cheng Jiang, ${ }^{\S}$ George K. Tofaris,* and Jason J. Davis* \\ Cite This: Anal. Chem. 2020, 92, 13647-13651 \\ Read Online
}

ABSTRACT: The egress of $\alpha$-synuclein in neuronally derived exosomes predates the clinical presentation of Parkinson's disease (PD), offering a means of developing a predictive or prognostic test. Here, we report the reagentless impedimetric assay of two internal exosome markers ( $\alpha$-synuclein and syntenin-1) from neuronal exosomes. Exosomes were efficiently extracted from patient sera

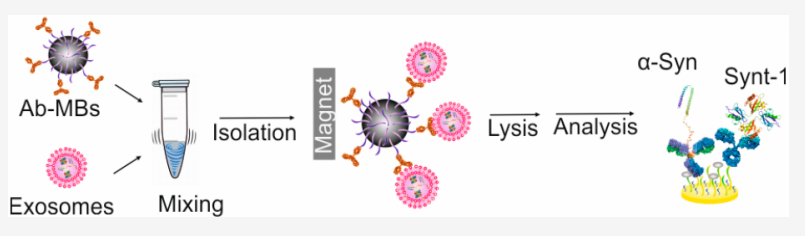
using anti-L1CAM conjugated zwitterionic polymer-modified magnetic beads prior to lysis and analyzed by electrochemical impedance spectroscopy. The quantification of $\alpha$-synuclein level across 40 clinical samples resolved statistically significant differences between PD patients and healthy controls (HC).

$\mathrm{P}$ arkinson's disease (PD) is the second most common neurodegenerative disease with increasing prevalence but, currently, no cure. It is primarily characterized by a movement disorder arising from a loss of dopaminergic neurons in the substantia nigra and intraneuronal $\alpha$-synuclein ( $\alpha$-Syn) aggregation. ${ }^{1} \mathrm{PD}$ is typically preceded by a long prodromal phase and can progress to dementia in advanced stages associated with diffuse $\alpha$-Syn aggregation throughout the brain. ${ }^{2,3}$ Currently, there is no blood or cerebrospinal fluid (CSF) biomarker in clinical practice for predicting PD in the prodromal phase or objectively assessing disease progression. The development of efficient methods to assay key biomarkers in the earliest stages of PD will undoubtedly underpin therapeutic intervention when disease-modifying therapies become available.

Exosomes are endosome-derived vesicles (40-140 nm) secreted by most cell types and detectable in most biological fluids including blood and CSF. ${ }^{4-6}$ They have been implicated in cell-cell signaling and the egress of unwanted proteins. We have very recently shown that the content of neuronally derived exosomes isolated from serum reflects pathological changes in the brain even at the prodromal stage of PD. ${ }^{4}$ However, neuron-derived exosomes constitute approximately $15 \%$ of total circulating exosomes ${ }^{4}$ and contain proteins at low concentrations requiring both effective extraction and a sensitive assaying of their contents. Exosomes are most typically isolated by differential ultracentrifugation (UC), a process which is laborious, equipment heavy, and associated with low isolation efficiency. ${ }^{7,8}$ Moreover, the standard differential ultracentrifugation methods do not discriminate between exosomes and other structures such as larger extracellular vesicles or protein/lipid aggregates. ${ }^{9,10}$ In recent years, immunoaffinity-based technology has been widely used to improve the efficiency and purity of exosome subtype isolation, and appropriately modified magnetic beads (MBs) have become a valuable part of this toolbox. ${ }^{11,12}$ They are, however, also readily fouled, and their "magnetic pull down" thus collects an unhelpfully low ratio of specific exosome to fouling material during immunocapture in complex biological fluids like serum, compromising the specificity of any downstream exosomal protein assay. ${ }^{13,14}$ This is especially troublesome for proteins such as $\alpha$-Syn which is present in biofluids primarily in a free form and in much lower abundance when exosome bound. ${ }^{2}$ The use of capture beads with higher levels of selectivity could mitigate this limitation. Of the nonfouling interfaces available, those based on highly hydrated zwitterionic polymers like poly(carboxybetaine methacrylamide)) (pCBMA) are the most potent. ${ }^{15}$ The most effective high density "brush type" interfaces are only realistically accessible through surface-initiated polymer growth, such as that through an appropriately designed reversible additionfragmentation chain transfer (RAFT). Once prepared, these films can be further modified with antibodies in generating specifically receptive interfaces. ${ }^{16,17}$

Conventional lab-based biomarker quantification is by enzyme-linked immunosorbent assay (ELISA); this is laborious, has high cost, and is often insufficiently sensitive. ${ }^{18}$ Electrochemical assays can be conveniently run with higher sensitivity, enhanced simplicity (single step and/or "reagentless"), and lower cost. ${ }^{19-22}$ There has been much interest in assessments of $\alpha$-Syn in PD from serum and CSF, though, thus far, these assessment have been of limited clinical value. ${ }^{23-25}$

Received: July 21, 2020

Accepted: September 18, 2020

Published: September 18, 2020 
Although a number of analyses of exosomes have been reported by electrochemical methods, these are almost exclusively of exosomes themselves and not internal protein markers. $^{26,27}$ We have recently reported the analysis of the internal cargo protein syntenin-1 (Synt-1) from UC-isolated serum exosomes. ${ }^{28}$ In this current study, polymer brush-coated magnetic beads were generated via RAFT and conjugated to anti-L1CAM (i.e., L1 cell adhesion molecule) antibodies for the specific immunocapture of neuronally derived exosomes. It was envisaged that improved levels of capture specificity would enable the use of comparatively simple sensor surfaces without detrimental loss of specificity. ${ }^{29-31}$ The isolation of exosomes was followed by lysis and a robust impedimetric quantification of two internal markers in a manner that enabled a statistically significant differentiation of PD from control samples.

\section{MAGNETIC BEAD CHARACTERIZATION AND EXOSOME ISOLATION}

As noted, nonspecific adsorption can negatively influence downstream exosomal proteins detection and analysis. To bypass this, magnetic beads $(\sim 2.4 \mu \mathrm{m})$ were coated with a zwitterionic polymer PCBMA via the RAFT process and were further modified with the anti-L1CAM antibody (Figure 1).

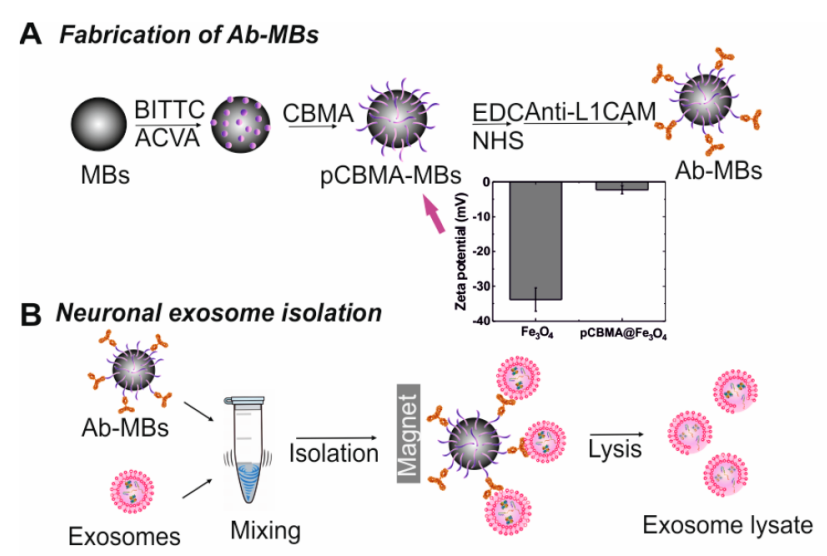

C Label-free impedance assay

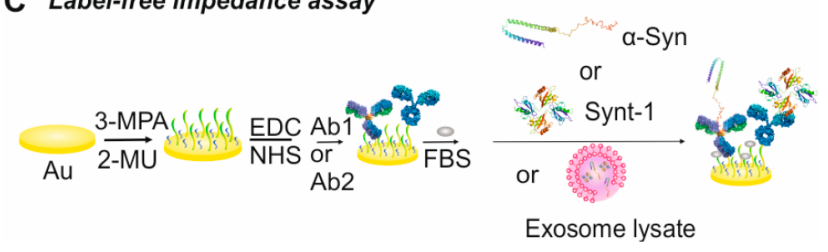

Figure 1. Schematic representation of (A) the synthesis of pCBMAcoated $\mathrm{MBs}$ and anti-L1CAM $\mathrm{Ab}$ conjugation. Inset shows zeta potential of bare $\mathrm{MBs}$ before (marked as $\mathrm{Fe}_{3} \mathrm{O}_{4}$ ) and after polymerization (pCBMA@ $\mathrm{Fe}_{3} \mathrm{O}_{4}$ ). (B) Neuronal exosome isolation using anti-L1CAM pCBMA-coated MBs. (C) Label-free impedimetric assays for two internal markers $(\mathrm{Ab} 1=$ anti- $\alpha$-Syn antibody, $\mathrm{Ab} 2=$ anti-Synt-1 antibody).

Successful polymerization of pCBMA on beads was resolved by infrared attenuated total reflection spectroscopy (IR-ATR) (Figure S1A). ${ }^{32,33}$ Zeta potential assessments (Figure 1A) were measured before $\left(\mathrm{Fe}_{3} \mathrm{O}_{4},-33.8 \pm 3.2 \mathrm{mV}\right)$ and after (pCBMA@ $@ \mathrm{Fe}_{3} \mathrm{O}_{4},-2.3 \pm 1.2 \mathrm{mV}$ ) polymerization, indicating a near-zero overall charge ${ }^{34,35}$ as desired for optimal performance. $^{36,37}$ The antifouling properties of the pCBMA@ $@ \mathrm{Fe}_{3} \mathrm{O}_{4}$ MBs were confirmed through a markedly reduced ( 90\%) nonspecific adsorption of bovine serum albumin (BSA) when compared to native $\mathrm{Fe}_{3} \mathrm{O}_{4}$ beads (Figure S1B) It is noteworthy that, even after antibody conjugation (i.e., anti-L1CAM modified pCBMA@ $\mathrm{Fe}_{3} \mathrm{O}_{4} \mathrm{MBs}$ ), antifouling performance is not significantly compromised. ${ }^{38,39}$ We further demonstrated that pCBMA@ $\mathrm{Fe}_{3} \mathrm{O}_{4} \mathrm{MBs}$, unlike commercially available carboxylate MBs, exhibited good antifouling properties when incubated with soluble recombinant $\alpha$-synuclein, irrespective of the antibody used (anti-L1CAM or anti-HA as shown in Figure $2 \mathrm{~A}$ ). This is critically important in supporting the selective and clean isolation of exosomes from serum samples.

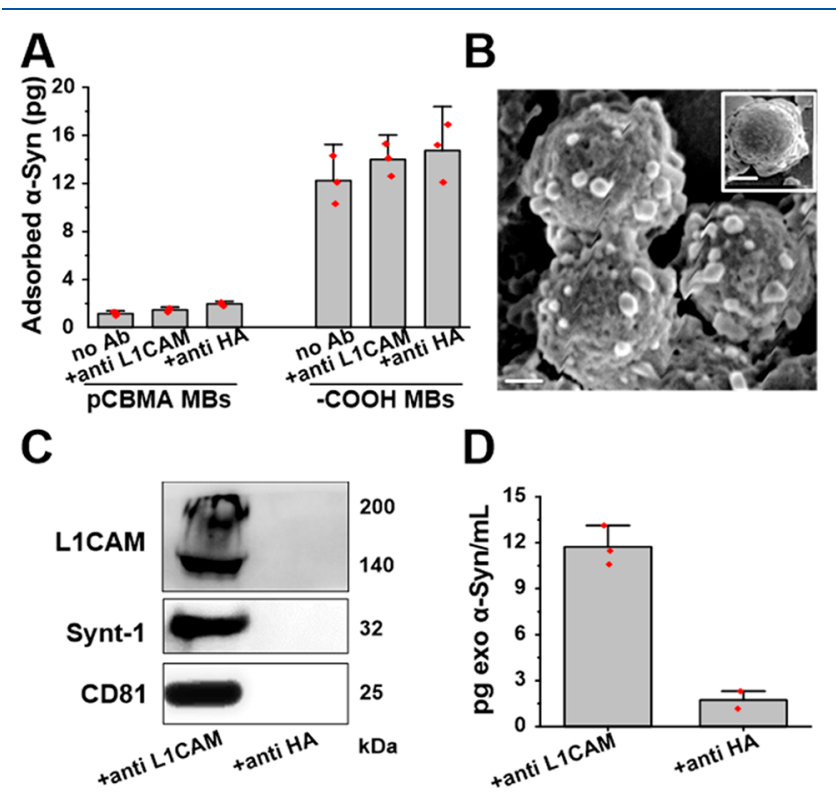

Figure 2. (A) Histogram depicting the quantified adsorption of recombinant $\alpha$-Syn on different Ab-modified pCBMA@ $@ \mathrm{Fe}_{3} \mathrm{O}_{4} \mathrm{MBs}$ surfaces. The commercial carboxylic acid-terminated $\mathrm{MBs}$ were used as the control. (B) SEM image of serum-captured exosomes on antiL1CAM-modified MBs versus anti-HA (control)-modified MBs (insert). Scale bar $1 \mu \mathrm{m}$. (C) Immunoblotting of lysates of immunocaptured vesicles confirming the detection of both transmembrane proteins (L1CAM, CD81) and internal protein Synt-1 from exosomes. Specific electrochemiluminescence detection of $\alpha$-Syn (D) in neuronal exosomes immunocaptured from serum with antiL1CAM vs anti-HA (control)-modified pCBMA@ $@ \mathrm{Fe}_{3} \mathrm{O}_{4} \mathrm{MBs}$.

The anti-L1CAM antibody-coated pCMBA were then assessed for immunocapture of neuronal exosomes in serum. SEM image analysis clearly showed exosomes bound to antiL1CAM conjugated pCBMA@ $\mathrm{Fe}_{3} \mathrm{O}_{4} \mathrm{MBs}$ (Figure 2B) but not control beads (i.e., anti-HA Ab-coated pCBMA@ $@ \mathrm{Fe}_{3} \mathrm{O}_{4}$ beads, inset in Figure 2B). Captured exosomes were eluted using a pH 2.9 glycine solution, followed by neutralization with a Tris buffer and negative staining for TEM (Figure S1C). The image resolves double-membrane and cup-shaped vesicles with a diameter of $\sim 100 \mathrm{~nm}$, typical of exosome morphology and size. ${ }^{40}$ To further confirm their molecular composition, captured vesicles were lysed and processed for immunoblotting (Figure 2C). The transmembrane markers L1CAM and CD 81 and the internal protein marker Synt-1 were detected in lysates from anti-L1CAM@pCBMA@ $\mathrm{Fe}_{3} \mathrm{O}_{4}$ MBs samples but not in control lysates (samples incubated with anti-HA-coated pCBMA@ $\left.\mathrm{Fe}_{3} \mathrm{O}_{4} \mathrm{MBs}\right)$.

We also confirmed that anti-L1CAM-modified pCBMA@ $\mathrm{Fe}_{3} \mathrm{O}_{4} \mathrm{MBs}$ are effective in isolating from serum neuronal exosomes containing $\alpha$-Syn (Figure 2D). 
It is noteworthy that the ratio of CD81 content in L1CAM+ (neuronal exosomes)/CD9+ (generic exosomes) was calculated to be $\sim 11 \%$ based on a comparative analysis of immunoblot intensities (Figure S2). Taken together, these observations strongly support the selective isolation of serum neuronal exosomes containing $\alpha$-Syn by anti-L1CAM-modified pCBMA@ $@ \mathrm{Fe}_{3} \mathrm{O}_{4}$ beads. The overall immunocapture efficiency was also examined using anti-CD9-modified pCBMA@ $@ \mathrm{Fe}_{3} \mathrm{O}_{4}$ (Figure S3) and resolved $81 \%$ by nanoparticle tracking analysis.

Subsequent to this analysis of selective exosome capture, we sought to quantify internal markers electrochemically. Mixed, low initial impedance and high impedimetric baseline stability, self-assembled monolayers (SAMs) comprising 3-mercaptopropionic acid (3-MPA), 2-mercaptoethanol (2-MU) were generated on gold electrodes prior to antibody loading (Figure S4). These interfaces were thereafter applied to the analysis of exosomal content (Figure 1C) after controlled lysis; specifically, both $\alpha$-Syn and Synt-1 were assayed. The former is associated with $\mathrm{PD},{ }^{3}$ whereas the latter, as prior indicated, is a generic cargo protein.

\section{LABEL-FREE IMPEDIMETRIC ASSAYS IN SPIKED SERUM}

Prior to the analysis of real patient samples, we initially set out to gauge the reliability of biomarker quantification through the repeat analysis of prepared spiked solutions for both $\alpha$-Syn and Synt-1, including analyses with control proteins (e.g., Creactive protein (CRP) and BSA) at greater than $10^{6}$ times excess of the expected marker levels (Figure S5). Reliable triplicate quantifications of both markers (Figure S6) were demonstrable within $30 \mathrm{~min}$ with limits of detection (LOD) and quantification (LOQ) at 0.3 and $0.8 \mathrm{pg} / \mathrm{mL}$ for $\alpha$-Syn, respectively (Figure S7A), notably better than most prior exosomal analyses. ${ }^{41}$ The assays herein are significantly more sensitive than commercial electrochemiluminescence kits (by almost an order of magnitude), much cheaper, much faster, and require markedly less sample input (100 vs $500 \mu \mathrm{L})$.

\section{CLINICAL SAMPLE ANALYSIS}

A statistical analysis across 40 clinical samples in a cohort study between PD and HC ( $n=20$ per group) was conducted to access the applicability of internal neuronal exosomal biomarkers to differentiate between PD and HC using $10 \mu \mathrm{L}$ of exosome lysate immunocaptured from $100 \mu \mathrm{L}$ of input serum using anti-L1CAM-coated pCBMA@ $@ \mathrm{Fe}_{3} \mathrm{O}_{4} \mathrm{MBs}$. Box plot analyses (Figure 3) confirmed an elevation of $\alpha$-Syn in neuronal exosomes in PD ( $p$ value of $4.3 \times 10^{-4}$, i.e., $\left.<0.0001\right)$. There was no difference in the content of the generic exosomal protein Synt-1 $(p=0.34)$ between the two groups. To further assess the reliability of the electrochemical assay in measuring neuronal exosome-associated $\alpha$-Syn and Synt-1, we cross referenced the same samples with the electrochemiluminescence kit platform (Figure S9) where the difference between $\mathrm{PD} v s \mathrm{HC}$ was also observed $\left(p=1.48 \times 10^{-5}\right.$ albeit with $500 \%$ greater serum volume). Absolute quantifications of $\alpha$-Syn using EIS were higher than those detected by electrochemiluminescence. This may be a reflection of differences in assay sensitivity and selectivity at the respective surfaces $\left(32 \mathrm{~mm}^{2}\right.$ in 96-well chemiluminescence plate $v s 9 \mathrm{~mm}^{2}$ in EIS sensor and different surface chemistries). ${ }^{42-44}$ To the best of our knowledge, this is the first time that a reagentless electro-
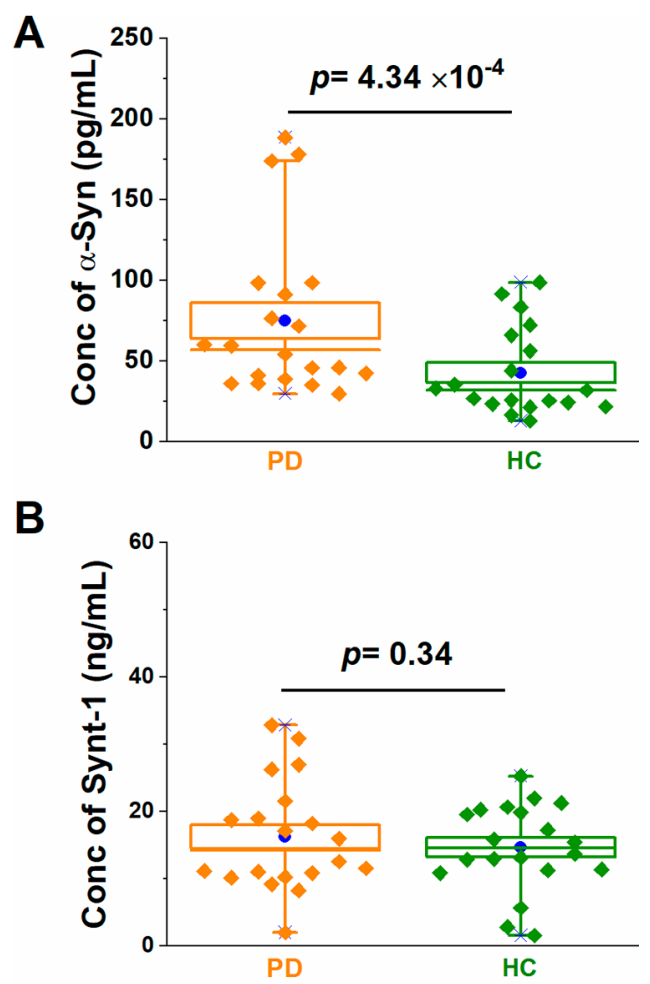

Figure 3. Box plots for impedimetric exosomal quantifications of (A) $\alpha$-Syn and (B) Synt- 1 from 40 clinical samples (PD and HC, $n=20$ per group). In the box plots, the lower and upper boundaries indicate the 25 th and 75 th percentiles, respectively. The line within the box marks the median, and the blue circle within the box marks the mean. Diamonds represent individual patient sample data points (the mean value of nine measurements across three electrodes for each clinical sample).

chemical method has been shown to be of clinical value in analyzing the protein content of exosomes.

\section{CONCLUSIONS}

Anti-L1CAM-modified polymer brush-coated MBs enable the selective immunocapture of neuronal exosome subpopulations from $100 \mu \mathrm{L}$ of serum prior to a sensitive (sub $\mathrm{pg} / \mathrm{mL}$ ) and reagentless impedimetric assay. The cleanliness of the initial isolation enables subsequent electroanalyses to be performed on a standard and readily scalable monolayer film from much lower levels of serum than required for electrochemiluminescence or ELISA. Significantly, these analyses further confirm the value of exosomal of $\alpha$-Syn as a relevant biomarker in PD. ${ }^{4}$ The study herein offers a solution for effectively verifying biomarkers in clinical translation and also lays a foundation for on-chip integration of immunocapture and reagentless analysis of exosome biomarkers in a scalable manner.

\section{ASSOCIATED CONTENT}

\section{Supporting Information}

The Supporting Information is available free of charge at https://pubs.acs.org/doi/10.1021/acs.analchem.0c03092.

Materials and methods, magnetic bead characterization, selectivity assessments, and supplementary data (PDF) 


\section{AUTHOR INFORMATION}

\section{Corresponding Authors}

George K. Tofaris - Nuffield Department of Clinical Neurosciences, University of Oxford, Oxford OX3 9DU, United Kingdom; Email: george.tofaris@ndcn.ox.ac.uk

Jason J. Davis - Department of Chemistry, University of Oxford, Oxford OX1 3QZ, United Kingdom; @ orcid.org/0000-00017734-1709; Email: jason.davis@chem.ox.ac.uk

\section{Authors}

Ying Fu - Department of Chemistry, University of Oxford, Oxford OX1 3QZ, United Kingdom

Cheng Jiang - Nuffield Department of Clinical Neurosciences, University of Oxford, Oxford OX3 9DU, United Kingdom

Complete contact information is available at: https://pubs.acs.org/10.1021/acs.analchem.0c03092

\section{Author Contributions}

Y.F. and C.J. performed experiments and analyzed data. G.K.T. and J.J.D. designed research and analyzed data. The manuscript was written with contributions from all authors. All authors have given approval to the final version of the manuscript.

\section{Author Contributions}

${ }^{\S} \mathrm{Y}$. Fu and C. Jiang contributed equally.

\section{Notes}

The opinions expressed and arguments employed herein do not necessarily reflect the official views of these funding bodies. The authors declare no competing financial interest.

\section{ACKNOWLEDGMENTS}

We thank Dr. Robert Hein for the synthesis of the CBMA monomer and helpful input and Dr. Franziska Hopfner for providing clinical samples. The work was funded by grants from the EPSRC (EP/M006204/1), the Selfridges Group Foundation, and the NIHR Oxford Biomedical Research Centre to G.K.T and J.J.D.

\section{REFERENCES}

(1) Chaudhuri, K. R.; Healy, D. G.; Schapira, A. H. Lancet Neurol. 2006, 5, 235-245.

(2) Tofaris, G. K. J. Parkinson's Dis. 2017, 7, 569-576.

(3) Tofaris, G. K.; Spillantini, M. G. Mov. Disord. 2005, 20, S37S44.

(4) Jiang, C.; Hopfner, F.; Katsikoudi, A.; Hein, R.; Catli, C.; Evetts, S.; Huang, Y.; Wang, H.; Ryder, J. W.; Kuhlenbaeumer, G.; Deuschl, G.; Padovani, A.; Berg, D.; Borroni, B.; Hu, M. T.; Davis, J. J.; Tofaris, G. K. J. Neurol., Neurosurg. Psychiatry 2020, 91, 720-729.

(5) Tomlinson, P. R.; Zheng, Y.; Fischer, R.; Heidasch, R.; Gardiner, C.; Evetts, S.; Hu, M.; Wade-Martins, R.; Turner, M. R.; Morris, J.; Talbot, K.; Kessler, B. M.; Tofaris, G. K. Ann. Clin. Transl. Neurol. 2015, 2, 353-361.

(6) Stuendl, A.; Kunadt, M.; Kruse, N.; Bartels, C.; Moebius, W.; Danzer, K. M.; Mollenhauer, B.; Schneider, A. Brain 2016, 139, 481494.

(7) Théry, C.; Amigorena, S.; Raposo, G.; Clayton, A. Curr. Protoc. Cell Biol. 2006, 30, 3.22.1-3.22.29.

(8) Li, P.; Kaslan, M.; Lee, S. H.; Yao, J.; Gao, Z. Theranostics 2017, 7,789 .

(9) Taylor, D. D.; Zacharias, W.; Gercel-Taylor, C. In Serum/Plasma Proteomics: Methods and Protocols; Humana Press: Totowa, NJ, 2011; pp 235-246.
(10) Zlotogorski-Hurvitz, A.; Dayan, D.; Chaushu, G.; Korvala, J.; Salo, T.; Sormunen, R.; Vered, M. J. Histochem. Cytochem. 2015, 63, 181-189.

(11) Cai, S.; Luo, B.; Jiang, P.; Zhou, X.; Lan, F.; Yi, Q.; Wu, Y. Nanoscale 2018, 10, 14280-14289.

(12) Fan, Z.; Yu, J.; Lin, J.; Liu, Y.; Liao, Y. Analyst 2019, 144, $5856-5865$.

(13) Clayton, A.; Court, J.; Navabi, H.; Adams, M.; Mason, M. D.; Hobot, J. A.; Newman, G. R.; Jasani, B. J. Immunol. Methods 2001, 247, 163-174.

(14) Van Andel, E.; De Bus, I.; Tijhaar, E. J.; Smulders, M. M. J.; Savelkoul, H. F. J.; Zuilhof, H. ACS Appl. Mater. Interfaces 2017, 9, 38211-38221.

(15) Jiang, C.; Wang, G.; Hein, R.; Liu, N.; Luo, X.; Davis, J. J. Chem. Rev. 2020, 120, 3852-3889.

(16) Kanyong, P.; Catli, C.; Davis, J. J. Anal. Chem. 2020, 92, 47074710.

(17) Kanyong, P.; Patil, A. V.; Davis, J. J. Annu. Rev. Anal. Chem. 2020, 13, 183-200.

(18) Zangar, R. C.; Daly, D. S.; White, A. M. Expert Rev. Proteomics 2006, 3, 37-44.

(19) Fu, Y.; Wang, N.; Yang, A.; Law, H. K. W.; Li, L.; Yan, F. Adv. Mater. 2017, 29, 1703787.

(20) Chen, L.; Fu, Y.; Wang, N.; Yang, A.; Li, Y.; Wu, J.; Ju, H.; Yaen, F. ACS Appl. Mater. Interfaces 2018, 10, 18470-18477.

(21) Fu, Y.; Liu, K.; Sun, Q.; Lin, B.; Lu, D.; Xu, Z.; Hu, C.; Fan, G.; Zhang, S.; Wang, C.; Zhang, W. Biosens. Bioelectron. 2014, 56, 258263.

(22) Baradoke, A.; Hein, R.; Li, X.; Davis, J. J. Anal. Chem. 2020, 92, $3508-3511$

(23) Aerts, M.; Esselink, R.; Abdo, W.; Bloem, B.; Verbeek, M. Neurobiol. Aging 2012, 33, 430. e1-430. e3.

(24) Chahine, L. M.; Stern, M. B.; Chen-Plotkin, A. Parkinsonism Relat Disord. 2014, 20, S99-S103.

(25) Bougea, A.; Stefanis, L.; Paraskevas, G. P.; Emmanouilidou, E.; Vekrelis, K.; Kapaki, E. Neurol Sci. 2019, 40, 929-938.

(26) Jeong, S.; Park, J.; Pathania, D.; Castro, C. M.; Weissleder, R.; Lee, H. ACS Nano 2016, 10, 1802-1809.

(27) Yadav, S.; Boriachek, K.; Islam, M. N.; Lobb, R.; M?ller, A.; Hill, M. M.; Al Hossain, M. S.; Nguyen, N. T.; Shiddiky, M. J. A. ChemElectroChem 2017, 4, 967-971.

(28) Li, Q.; Tofaris, G. K.; Davis, J. J. Anal. Chem. 2017, 89, 31843190.

(29) Jiang, C.; Alam, M. T.; Parker, S. G.; Darwish, N.; Gooding, J. J. Langmuir 2016, 32, 2509-2517.

(30) Jiang, C.; Tanzirul Alam, M.; Parker, S. G.; Gooding, J. J. Electroanalysis 2015, 27, 884-889.

(31) Jiang, C.; Alam, M. T.; Silva, S. M.; Taufik, S.; Fan, S.; Gooding, J. J. ACS Sens 2016, 1, 1432-1438.

(32) Jiang, C.; Xu, S.; Zhang, S.; Jia, L. Anal. Biochem. 2012, 420, $20-25$.

(33) Dong, C.; Wang, H.; Zhang, Z.; Zhang, T.; Liu, B. J. Colloid Interface Sci. 2014, 432, 135-143.

(34) Chen, K.; Hu, F.; Gu, H.; Xu, H. J. Mater. Chem. B 2017, 5, 435-443.

(35) Ding, F.; Yang, S.; Gao, Z.; Guo, J.; Zhang, P.; Qiu, X.; Li, Q.; Dong, M.; Hao, J.; Yu, Q.; Cui, J. Front. Chem. 2019, 7, 770.

(36) Vísová, I.; Vrabcová, M.; Forinová, M.; Zhigunová, Y.; Mironov, V.; Houska, M.; Bittrich, E.; Eichhorn, K.-J.; Hashim, H.; Schovánek, P.; Dejneka, A.; Vaisocherová-Lísalová, H. Langmuir 2020, 36, 8485-8493.

(37) Keefe, A. J.; Jiang, S. Nat. Chem. 2012, 4, 59-63.

(38) Liu, S.; Jiang, S. Nano Today 2016, 11, 285-291.

(39) Yang, W.; Sundaram, H. S.; Ella, J.-R.; He, N.; Jiang, S. Acta Biomater. 2016, 40, 92-99.

(40) Fan, Z.; Xiao, K.; Lin, J.; Liao, Y.; Huang, X. Small 2019, 15, 1903761.

(41) An, Y.; Tang, L.; Jiang, X.; Chen, H.; Yang, M.; Jin, L.; Zhang, S.; Wang, C.; Zhang, W. Chem. - Eur. J. 2010, 16, 14439-14446. 
(42) Noble, J.; Knight, A.; Reason, A.; Di Matola, A.; Bailey, M. Mol. Biotechnol. 2007, 37, 99-111.

(43) Christiansson, L.; Mustjoki, S.; Simonsson, B.; OlssonStrömberg, U.; Loskog, A. S.; Mangsbo, S. M. EuPa Open Proteomics 2014, 3, 37-47.

(44) Kinn Rød, A. M.; Harkestad, N.; Jellestad, F. K.; Murison, R. Sci. Rep. 2017, 7, 6748. 